\title{
¿Túnica o Capa? Análisis Histórico, Etimológico y Propuesta para Terminologia Anatomica Internacional
}

\author{
¿Tunic or Coat? Historical, Etymological Analysis and \\ Proposal for the International Anatomical Terminology
}

Francisco Pérez-Rojasi'; Pablo Bustamante-Aliste ${ }^{2}$ \& Ricardo S. Puebla Wuth ${ }^{3}$

PÉREZ-ROJAS, F.; BUSTAMANTE-AliSTE, P. \& PUEBLA-WUTH, R. S. ¿Túnica o Capa? Análisis histórico, etimológico y propuesta para Terminologia Anatomica Internacional. Int. J. Morphol., 36(1):284-289, 2018.

RESUMEN: El continuo desarrollo de las ciencias morfológicas y la utilización de sus productos por diversas ciencias aplicadas ha provocado la incorporación a Terminologia Anatomica (TA) de modificaciones que tienden a interpretaciones de los nombres, que son dados por principio en latín como idioma oficial. Esta situación, hacen necesarias revisiones periódicas de la nomenclatura, sugiriendo adecuaciones confiables y válidas a la traducción al idioma vernáculo de los términos anatómicos. En este sentido, revisamos los términos en Terminologia Anatomica Internacional (TAI) de: Tunica mucosa linguae (A05.1.04.011), Tunica serosa (A05.4.01.007), Tunica adventitia (A05.4.01.009), Tunica muscularis (A05.4.01.010), Tunica mucosa (A05.4.01.015) у Tunica mucosa oris (A05.1.01.002); los que, traducidos al español por la Sociedad Anatómica Española (2001) se identifican como: mucosa de la lengua, capa serosa, capa adventicia, capa muscular, capa mucosa y mucosa de la boca, respectivamente; reconociendo, errores etimológicos, en la traducción del término Túnica (en latin: Tunica) por el de Capa (del latín Cappa) o su ausencia. Evidenciado esto, realizamos un análisis de los términos latinos Tunica y Cappa, y su traducción al español, encontrando: inconsistencias en la traducción de los términos, falta de traducción de la palabra Tunica; y, la verificación fundamentada de tales inconsistencias en la etimología de los términos descritos. A la luz de los resultados, basándose en los principios de la TA y el principio de construcción del cuerpo humano de estratificación, sugerimos: cambiar la traducción en TAI para los términos Tunica mucosa linguae, Tunica serosa, Tunica adventitia, y Tunica mucosa oris por: Mucosa linguae, Cappa serosa, Cappa adventitia, Mucosa oris; y, cambiar, la traducción al español de los términos Tunica muscularis y Tunica mucosa hacia Túnica muscular y Túnica mucosa, respectivamente. El uso de términos anatómicos correctos permitirá una mejor comunicación en el ámbito de la docencia y la investigación científica.

PALABRAS CLAVE: Túnica; Capa; Terminologia Anatomica.

\section{INTRODUCCION}

El estudio, utilización y manejo de la Anatomía Humana está vinculada desde su origen al ejercicio mismo de las ciencias aplicadas, desde las ciencias médicas y la robótica hasta la antropología física, pueden constatarse una gran diversidad de ámbitos de utilización para el uso de los términos anatómicos. Dada esta situación, se han acuñado conceptos diversos referidos a estructuras anatómicas, que no siguen estrictamente la utilización de nombres con la rigurosidad con que Terminologia Anatomica Internacional (TAI) cuida que se maneje la clasificación anatómica: utilizar el latín como referente de idioma oficial; asignar un único nombre por término, otorgándole valor descriptivo a este; y, propender a la eliminación de epónimos (Vásquez \& del Sol, 2014; Roa et al., 2016; Vargas et al.,
2016). De hecho, se sabe que muchos términos o nombres son usados por diferentes autores, y con cierto grado de detalle, no es claro qué término se refiere a qué concepto (Grkovic et al., 2009). Esto, hace que el interés y cuidado por establecer una terminología común sea cada vez más imperativo y necesario, en aras de aunar los distintos esfuerzos por establecer y mantener un lenguaje universal a lo largo del tiempo para definir las estructuras que conforman el cuerpo humano (Álvarez, 2007); y, sigue, siendo una tarea clave para ordenar el lenguaje científico posibilitando un intercambio fluido de información, lo que se proyecta como una labor de relevancia sobre todo para homogeneizar el lenguaje en la práctica clínica (Kachlik et al., 2009). Todo lo anterior, sigue siendo muy complejo por el

\footnotetext{
${ }^{1}$ Escuela de Medicina, Universidad Autónoma de Chile, Talca, Chile.

${ }^{2}$ Departamento de Ciencias Morfológicas, Facultad de Ciencia, Universidad San Sebastián, Puerto Montt, Chile.

${ }_{3}^{3}$ Docente Programa de Magíster en Neurociencias de la Educación, Escuela de Postgrado, Universidad Mayor, Temuco, Chile.
} 
gran avance del conocimiento científico en todas las áreas de la Morfología Humana, lo que hace aún más necesario la búsqueda de un vocabulario común y reconocido a nivel internacional (Vásquez \& del Sol, 2014). Este ha sido el objetivo principal de los anatomistas y morfólogos desde fines del siglo XIX, cuando se comenzaron a revisar todos los términos anatómicos que venían siendo usados hasta ese momento, y se elaboró la primera Nómina Anatómica en latín, en Basilea en el año 1895.

Luego, las referencias indican que en el año 2009 se iniciaron los Simposios Ibero-Latinoamericanos de Terminologia Anatomica, Histologica y Embryologica (SILAT), cuya tarea es revisar las terminologías latinas y sugerir su traducción a los idiomas español y portugués. En el 2011, se presentó la segunda edición de Terminologia Anatomica Internacional. La edición fue elaborada por el FIPAT, Programa Internacional Federativo en Terminologia Anatomica, que reemplazó al FCAT (FCAT, 2000; FIPAT, 2011). La versión en español de Terminologia Anatomica Internacional fue elaborada en conjunto por la IFAA y la Sociedad Anatómica Española. Fue publicada en el año 2001, y es la única edición en español de Terminologia Anatomica Internacional (FCAT, 2001).

La revisión de los orígenes históricos, que apoya el uso de la terminología, nos permite comprender la complejidad que existe en el establecimiento de una Terminologia Anatomica común; y, como, luego de 122 años de la publicación del primer consenso terminológico, como fue Nomina Anatomica de Basilea, continua siendo necesario hacer revisiones y adecuaciones a los términos anatómicos, especialmente al momento de realizar su traducción al idioma vernáculo y por la gran cantidad de publicaciones internacionales referentes al área, para establecer una terminología en común, debido al elevado interés por el estudio de la Anatomía y su aplicación en diversas prácticas quirúrgicas y clínicas, ayudando a relacionar conceptos con su ubicación, forma y función en el cuerpo humano, a diferencia de lo que ocurría con los epónimos. Para Vesalio, la nomenclatura y sus términos debían ser simples y sencillos para que pudieran recordarse con facilidad mediante su asociación con cosas de la vida cotidiana (Vásquez \& del Sol op. cit.).

De lo anteriormente señalado, se analiza en este trabajo el término Tunica el que es asignado a una gran cantidad de estructuras anatómicas para definir su correspondiente término latino, y es traducido de manera inconsistente al español como capa (Tabla I).

El objetivo de este trabajo consiste en un análisis del significado del término latín Tunica, revisar su traducción al español como capa y discutir si su inconsistencia en la traducción mantiene algún fundamento etimológico, sugiriendo la revisión de los términos latinos Tunica y Cappa, y su ausencia de traducción al español (Tabla II). Se identifica el conflicto en la traducción del término latino Tunica como capa y se realiza un estudio etimológico histórico, para resolver esta dicotomía, es decir, verificar el término indicado respecto a la estructura: Tunica o Cappa.

\section{MATERIAL Y MÉTODO}

Se efectuó una revisión de artículos científicos, diccionarios históricos, Diccionario de Latín Oxford 1968, Diccionario de la Real Academia Española de la Lengua (RAE, 2017), Diccionario médico-biológico, histórico y etimológico de Salamanca y de las terminologías anatómicas: Terminologia Anatomica Internacional, del año 2001, publicada por la IFAA y la Sociedad Anatómica Española, con traducción al español, como así también la "International Anatomical Terminology", publicada por la FIPAT, en el año 2011, correspondiendo únicamente a la traducción en inglés de los términos en latín.

Tabla I. Ejemplos de traducción de Tunica al español.

\begin{tabular}{lll}
\hline Código de Terminología & Raíz Latina & Español \\
\hline A05.4.01.007 & Tunica serosa & Capa serosa \\
A05.4.01.009 & Tunica adventitia & Capa adventicia \\
A05.4.01.010 & Tunica muscularis & Capa muscular \\
A05.4.01.015 & Tunica mucosa & Capa mucosa \\
\hline
\end{tabular}

Tabla II. Ejemplos de ausencia de traducción de Tunica al español.

\begin{tabular}{lll}
\hline Código de Terminología & Raíz Latina & Español \\
\hline A05.1.04.011 & Tunica mucosa linguae & Mucosa lingual \\
A05.1.01.002 & Tunica mucosa oris & Mucosa de la
\end{tabular}




\section{RESULTADOS}

En 1895, en Basilea, Suiza, se establecieron los principios rectores de Nomina Anatomica (BNA) de los cuales: Universalidad, respetar en lo posible el vocablo en latín y su traducción al idioma vernáculo; Describir el elemento, de la forma más fiel a los principios de la geometría y la forma; y, Congruencia del término, o sea si se aceptan sus derivados, se debe aceptar la palabra primitiva (Cruz et al., 2010b). De estos principios se puede establecer que existen inconsistencias en la denominación latina de Tunica y la traducción al español que figura como Capa.

Para hacer un análisis de los orígenes del uso de los términos Tunica y Cappa, a través del sitio web del Diccionario Médico de la Universidad de Salamanca (Universidad de Salamanca, 2014), se accedió a su significado; "Tunica" Lexema: tunica lat. tunica $(\mathrm{m})$ "túnica" túnica [tunica, tunic] f. (Anat.) Capa o membrana que cubre una parte del cuerpo. [tunica $(\mathrm{m})$ lat. 'túnica'] Leng. base: lat. Antigua calco del gr. Docum. en 1495 en esp. Docum. en lat. mediev. y en fr. en el s. XIV en sentido anatómico. "Cappa" Lexema: capa lat. cappa (m) 'capa' capa [layer, coat] f. (Anat.) Estructura laminar que se superpone; ej. capa córnea, capa germinal. [cappa $(\mathrm{m})$ lat. 'capa'] Leng. base: lat. Antigua. Docum. en 1008 en esp.

Ambos términos presentan una raíz latina, el primero con su base en latín clásico y la segunda desde el latín medieval.

$\mathrm{Al}$ realizar la búsqueda de ambas palabras en el diccionario de la RAE evidenciamos lo siguiente: Tunica: Del lat. tunica. 1. f. Vestidura de lana que usan algunos religiosos debajo de los hábitos. 2. f. Vestidura con o sin mangas, que usaban los antiguos y les servía como de camisa. 3. f. Amat. Membrana delgada que cubre algunas partes del cuerpo. Las túnicas de los ojos, de las venas. Cappa: Del lat. mediev. cappa "capa", 'capa con capucha', y este del lat. tardío cappa 'capucha'. 1. f. Prenda de vestir larga y suelta, sin mangas, abierta por delante, que se lleva sobre los hombros encima del vestido. 2. f. Aquello que cubre o baña alguna cosa. Inmediatamente se puede evidenciar la diferencia en el significado de cada término, donde túnica designa una membrana o vestidura que se posiciona bajo algo; en cambio, capa refiere a una prenda de vestir que se posiciona sobre otra estructura o encima.

Además, a través del nuevo tesoro lexicográfico de la RAE, se pesquisaron los términos en español buscando su raíz latina, accediendo a libros antiguos digitalizados, mediante el apartado documentación histórica de Túnica y de Capa. Se obtuvieron registros de libros antiguos desde 1495 a 1852, donde el significado en el contexto lexicográfico de estos términos sugería una interpretación distinta para ambos.

Las palabras por fecha "Antigua" son aquellas que se vienen usando en el vocabulario científico desde la antigüedad (s. V a.C. a s. V d.C.) hasta nuestros días de forma ininterrumpida, en unos casos por tratarse de palabras que se integraron en el léxico patrimonial del latín (es decir, el que pasó de padres a hijos de forma oral y sufrió una evolución fonética que se atiene a leyes fijas que permiten predecir con pequeño margen de error el resultado de esa evolución), en otros, por ser palabras que se transmitieron a través de escritos científicos (principalmente médicos) en cuyo caso: o no sufrieron grandes alteraciones o, si se han producido (más por cuestiones gráficas que fonéticas), no se atienen a leyes fijas. Las palabras griegas que pertenecen a este grupo se integraron al latín, en unos casos en época clásica (s. II a.C. a s. II d.C.), en otros en época tardía (s. III d.C. a s. IX. d. C.) o en época medieval (s. IX a s. XV) y, pueden documentarse en alguna de esas épocas. En los casos en que se documentan sólo en francés o español medievales, el Diccionario Médico de la Universidad de Salamanca considera que necesariamente tuvieron que existir en latín medieval, aunque no se pueda probar documentalmente (Universidad de Salamanca).

Respecto a los libros antiguos, los términos Tunica y Cappa fueron utilizados en diversos contextos, desde textos religiosos hasta obras populares, en los cuales se pudo confirmar la utilización del término Tunica como una membrana o vestidura interior y Cappa como una envoltura externa denominada "Capa" en español. A continuación, citamos las frases encontradas en los distintos diccionarios antiguos para el término "Tunica" (Figs. 1-5) y, para el término "Сappa" (Figs. 6-13).

\section{Tunica.}

Diccionario Casas 1570: Camisa, Tonga, Túnica (Fig. 1).

\section{Tunica. camicis, tonega, tunica.}

Fig. 1. Diccionario Casas, 1570.

Diccionario Academia de Autoridades (S-Z) 1739: Significa también aquella telilla, o película, que en algunas frutas está pegada a la cáscara, y cubre inmediatamente la carne (Fig. 2).

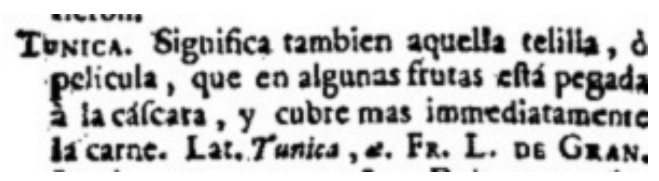

Fig. 2. Diccionario Academia de Autoridades (S-Z), 1739. 
Diccionario Academia Usual 1783: La membrana sutil, que cubre algunas partes del cuerpo; como: las Túnicas de los ojos, de las venas, etc (Fig. 3).

xúnica. La membrana sutil, que cubre algunas partes del cuerpo; como: las rúnicas de los ojos, de las venas, \&c. Tunica.:

Fig. 3. Diccionario Academia Usual, 1783.

Diccionario Terreros y Pando (P-Z), 1788: Los ojos están compuestos de muchas túnicas (Fig. 4).

del cuerpo. Fr. Tunique. Lat. Tunica. It. Tunica, membrana. Los ojos están compuestos de muchas túnicas.

Fig. 4. Diccionario Terreros y Pando (P-Z), 1788.

Diccionario Academia Usual 1869: Vestidura interior sin mangas que usaban los antiguos, y les servía como de camisa; La vestidura de lana interior que usaban los religiosos debajo de los hábitos (Fig. 5).

TUNICA. f. Vestidura interior sin mangas que usaban los antiguos, y les servia como de camisa. II La vestidura de lana que usan los religiosos debajo de los hábitos. || Vestidura exterior amplia y larga. ll La telilla $\delta$ película que en algunas frutas está pegada á la cáscara, y cubre más inmediatamente la carne. I La membrana sutil que cubre algunas partes del cuerpo; como: las túnicas de los ojos, de las venas, etc.

Fig. 5. Diccionario Academia Usual, 1869

\section{Cappa.}

Diccionario Nebrija 1495: Vestidura común (Fig. 6).

\section{Capa vefidiara comun.penula.ę.}

Fig. 6. Diccionario Nebrija, 1495.

Diccionario Alcalá 1505: Vestidura (Fig. 7).

\section{O2̉lpa veftioura区ăppa جuipăp.}

Fig. 7. Diccionario Alcalá, 1505.

Diccionario de Covarrubias 1611: La vestidura que se pone por sobre todas las demás (Fig. 8).

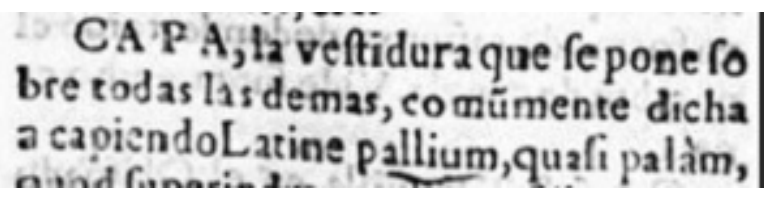

Fig. 8. Diccionario Covarrubias, 1611.
Diccionario Academia de Autoridades 1729: “...por lo cual se pone sobre todos los demás vestidos, y para adorno y seguridad tiene por." (Fig. 9).

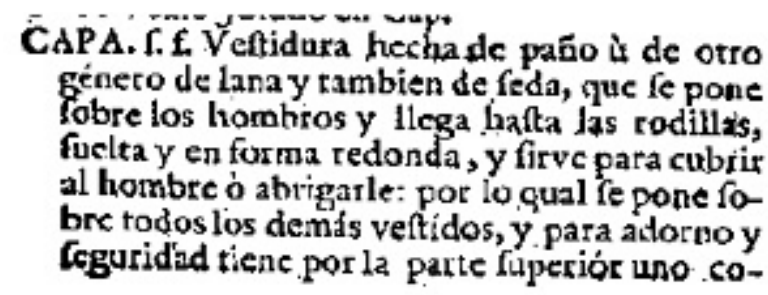

Fig. 9. Diccionario Academia de Autoridades, 1729.

Diccionario Academia Usual 1780: Lo que se echa por encima de otras cosas para cubrirlas (Fig. 10).

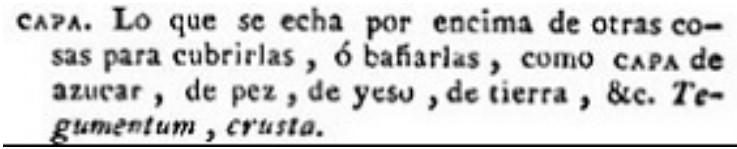

Fig. 10. Diccionario Academia Usual, 1780.

Diccionario Academia Usual 1783: Algunas veces se toma por la cubierta que se pone a las cosas para que no se maltraten, Operimentum, tegumentum. (Fig. 11).

\section{CAPA. Algunas veces se toma por la cubierta que - se pone á las cosas para que no se maltraten. Operimentum, tegumentum.}

Fig. 11. Diccionario Academia Usual, 1783.

Diccionario Academia Usual 1817: Lo que se hecha por encima de otras cosas para cubrirlas o bañarlas, como capa de azúcar, de pez, de yeso, de tierra, etc. Tegumentum, crusta (Fig. 12).

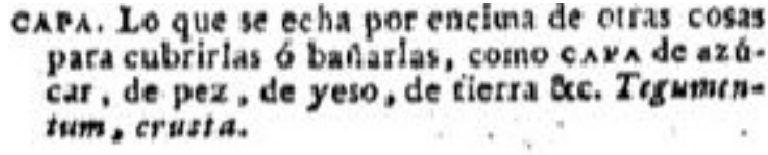

Fig. 12. Diccionario Academia Usual, 1817.

Diccionario Academia Usual 1852: Es también la parte superficial de una cosa. Así se dice una capa de tierra (Fig. 13).

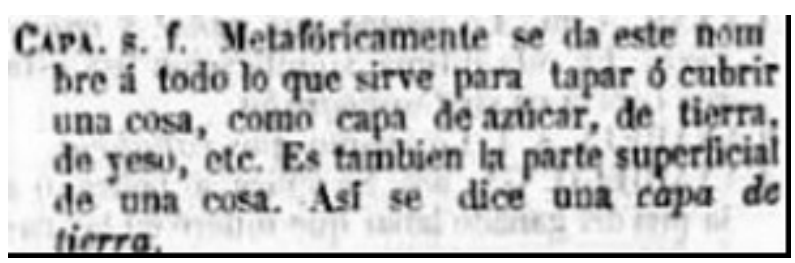

Fig. 13. Diccionario Academia Usual, 1852. 
Por último, al revisar el uso de ambos términos en diccionarios antiguos, se pudo evidenciar que a partir del año 1620 en el Diccionario Franciosini B, se ocupa la palabra Cappa en la explicación del significado del término "túnica" de esta forma "cappa da battuti" (Fig. 14). El término túnica es, igualmente, definido como "vestido exterior talar. Tunica talaris." en el Diccionario Academia Usual de 1783 (Fig. 15). Y, se observa una generalización del término túnica por simplificación de explicaciones en el Diccionario Terreros y Pando de 1788 (Fig. 16), como "En la Anatomía se llama también túnica la membrana delgada que cubre algunas partes del cuerpo". En los años sucesivos, ambos términos se hicieron homólogos.

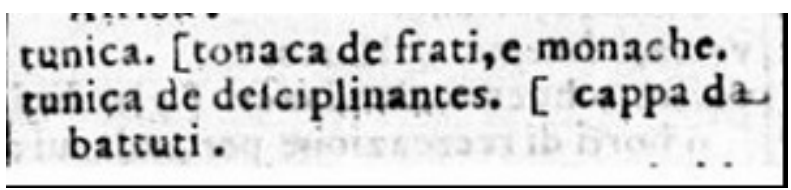

Fig. 14. Diccionario Franciosini B, 1620.

\section{T6wica. Vestido exterior talar. Tunica talaris.}

Fig. 15. Diccionario Academia Usual, 1783.

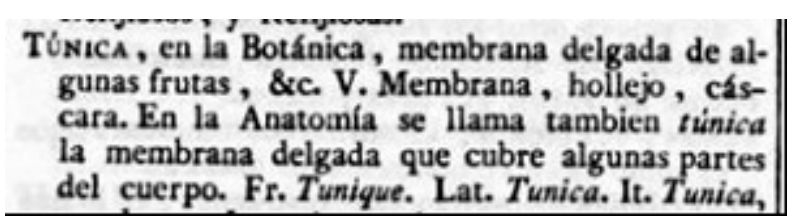

Fig. 16. Diccionario Terreros y Pando, 1788.

\section{DISCUSIÓN}

Existen problemas con algunos términos imprecisos que se utilizan en la práctica quirúrgica profesional y también con las numerosas denominaciones que se le atribuyen a una misma estructura en publicaciones médicas actuales (Grinberg, 1999; Duque Parra et al., 2002; JiménezGutiérrez, 2009; Vogl, 2009; Matusz, 2010; Cantín \& Suazo Galdames, 2011; Vandaele \& Gingras Harvey, 2013; Vargas et al., 2016; Losardo et al., 2015).

Al revisar los términos Tunica mucosa linguae (A05.1.04.011), Tunica serosa (A05.4.01.007), Tunica adventitia (A05.4.01.009), Tunica muscularis (A05.4.01.010), Tunica Mucosa (A05.4.01.015) y Tunica Mucosa oris (A05.1.01.002) se observa que en la traducción al español por la Sociedad Anatómica Española, se iden- tifican como: mucosa de la lengua, capa serosa, capa adventicia, capa muscular, capa mucosa y mucosa de la boca, respectivamente. Sin embargo, los hallazgos remitidos en los resultados demuestran una clara debilidad en cuanto al significado de cada concepto. Es posible constatar, que los términos Tunica y Cappa, presentan inconsistencias que conviene mejorar, puesto que etimológicamente son conceptos distintos. Tanto la traducción del término Tunica, que terminó homologándose a una cobertura que cubre algunas partes del cuerpo, desconociendo en el tiempo su significado como membrana o cubierta interna, como el de Cappa, que refiere claramente a la envoltura externa, que se posiciona sobre los vestidos, lo que se echa por encima de otras cosas o la parte superficial de una cosa. En relación con las evidencias presentadas, podemos demostrar que ambos términos en un principio eran acepciones totalmente distintas, donde Tunica refería a una envoltura interna y Cappa a una externa. Esta diferencia queda establecida muy claramente en el Diccionario Academia de Autoridades, 1739 para la palabra Tunica y en el Diccionario de Covarrubias, 1611, para la palabra Cappa. Debido a lo anterior, proponemos la adecuación de los términos "Tunica" y "Cappa" a Terminologia Anatomica Internacional, respetando el significado etimológico de ambos términos. Por tanto, túnica no debiera ser traducido como capa al español.

Por otra parte, al revisar el uso de ambos términos en diccionarios antiguos, se pudo demostrar que a partir del año 1620 en el Diccionario Franciosini B, se ocupa la palabra Cappa en la explicación del significado del término "tunica", de esta forma "cappa da battuti" (Fig. 14). El término túnica es, igualmente, definido como "vestido exterior talar. Tunica talaris." en el Diccionario Academia Usual de 1783 (Fig. 15). Y, se observa una generalización del término túnica por simplificación de explicaciones en el Diccionario de Terreros y Pando de 1788 (Fig. 16) como "En la Anatomía se llama también túnica la membrana delgada que cubre algunas partes del cuerpo". En los años sucesivos, ambos términos se hicieron homólogos haciéndose común referirse a una o la otra indistintamente.

Así también, al observar los términos Tunica тисоsa linguae (A05.1.04.011) y Tunica mucosa oris (A05.1.01.002) estimamos que estos presentan un error por omisión en su traducción al español al eliminar la palabra Tunica, quedando simplemente estos como "Mucosa lingual" y "Mucosa Oral" respectivamente. Por tanto, para recuperar la originalidad de ambos términos, proponemos: Mucosa linguae para Tunica mucosa linguae у Mucosa oris para Tunica тисоsa oris.

Por último, basándose en Terminologia Anatomica y el principio de construcción del cuerpo humano de "estra- 
tificación", sugerimos cambiar la traducción en Terminologia Anatomica para los términos: Tunica mucosa linguae, Tunica serosa, Tunica adventitia, Tunica mucosa oris por: Mucosa linguae, Cappa serosa, Cappa adventitia, Mucosa oris. Y, cambiar, la traducción al español de los términos Tunica muscularis y Tunica mucosa que fueron mal traducidos como: "Capa Muscular" y "Capa Mucosa", por: Túnica Muscular y Túnica Mucosa, respectivamente.

El análisis histórico, etimológico y la propuesta para las palabras "Tunica y Cappa", debieran ser ampliado y extensivo para los términos anatómicos que presenten este tipo de homologación indebida. El uso de términos anatómicos correctos permitirá una mejor comunicación en el ámbito de la docencia y la investigación científica.

PÉREZ-ROJAS, F.; BUSTAMANTE-ALISTE, P. \& PUEBLAWUTH, R. S. Tunic or Coat? Historical, etymological analysis and proposal for the International Anatomical Terminology. Int. J. Morphol., 36(1):284-289, 2018.

SUMMARY: The continuous development of the morphological sciences and the use of their products by various applied sciences has led to the incorporation of modifications into Anatomical Terminology (TA) that tend to interpretations of the names, which are given by principle in Latin as the official language. This situation requires periodic reviews of the nomenclature, suggesting reliable and valid adjustments from the translation into the vernacular language of the anatomical terms. In this sense, we review the terms in International Anatomical Terminology (IAT) of: Tunica mucosa linguae (A05.1.04.011), Tunica serosa (A05.4.01.007), Tunica adventitia (A05.4.01.009), Tunica muscularis (A05.4.01.010), Tunica mucosa (A05.4.01.015) and Tunica mucosa oris (A05.1.01.002); those that, translated into Spanish by the Spanish Anatomical Society (2001) are identified as: mucosa of the tongue, serosa layer, adventitial layer, muscle layer, mucous layer and mucosa of the mouth, respectively; recognizing, etymological errors, in the translation of the term Tunica (in Latin: Tunica) by Capa (from the Latin Cappa) or its absence. Evidenced this, we made an analysis of the Latin terms Tunica and Cappa, and its translation into Spanish, finding: inconsistencies in the translation of the terms, lack of translation of the word Tunica; and, the substantiated verification of such inconsistencies in the etymology of the described terms. In the light of the results, based on the principles of the TA and the principle of construction of the human body of "stratification", we suggest: change the translation in TAI for the terms Tunica mucosa linguae, Tunica serosa, Tunica adventitia, and Tunica mucosa oris by: Mucosa linguae, Cappa serosa, Cappa adventitia, Mucosa oris, and, change, the translation into Spanish of the terms Tunica muscularis and Tunica mucosa into Muscular Tunic and Tunic Mucosa, respectively.The use of correct anatomical terms will allow better communication in the field of teaching and scientific research.

KEY WORDS: Tunic; Coat; Terminologia Anatomica.

\section{REFERENCIAS BIBLIOGRÁFICAS}

Álvarez, A. Textos Sociolingüísticos. Mérida, Editorial Universidad de Los Andes, 2007.

Cantín, M. \& Suazo Galdames, I. Cleft lip in biomedical terminology. Rev. Med. Chile, 139(2):274-5, 2011.

Duque Parra, J. E.; Gómez Arias, N. C. \& Giraldo, R. D. P. Nomenclatura Anatómica Internacional. Med. U. P. B., 21(1):43-55, 2002.

Grinberg, M. Epônimos em Cardiologia. Homenagem e exemplo. São Paulo, Livraria Roca, 1999.

Grkovic, I.; Marinovic Guic, M., Kosta, V.; Poljicanin, A.; Caric, A. \& Vilovic, K. Designing anatomy program in modern medical curriculum: matter of balance. Croat. Med. J., 50(1):49-54, 2009.

Jiménez-Gutiérrez, I. La sinonimia y la polisemia en la terminología anatómica: términos de ubicación y de relación de estructuras anatómicas. Entreculturas, 1:579-97, 2009.

Kachlik, D.; Bozdechova, I.; Cech, P.; Musil, V. \& Baca, V. Mistakes in the usage of anatomical terminology in clinical practice. Biomed. Pap. Med. Fac. Univ. Palacky Olomouc Czech Repub., 153(2):157-61, 2009.

Losardo, R. J.; Prates, N. E. V. B.; Arteaga-Martínez, M.; Cabral, R. H. \& García-Peláez, M. I. International Morphological Terminology: More than anatomy, histology and embryology. Int. J. Morphol., 33(1):4007, 2015.

Matusz, P. Misleading Latin/English equivalents for some liver terms in Terminologia Anatomica. Anat. Sci. Educ., 3(3):156-7, 2010.

Real Academia Española (RAE). Nuevo Tesoro Lexicográfico de la Lengua Española. Madrid, Real Academia Española, 2017. Disponible en: http://ntlle.rae.es/ntlle/SrvltGUILoginNtlle

Roa, I.; Vásquez, B. \& Contreras, M. Eponyms persistence in Terminologia Histologica. Int. J. Morphol., 34(4):1245-52, 2016.

Sociedad Anatómica Española (SAE). Terminologia Anatomica. Madrid, Médica Panamericana, 2001.

Universidad de Salamanca. Diccionario Médico-Biológico, Histórico y Etimológico. Salamanca, Ediciones Universidad de Salamanca, 2014. Disponible en: http://dicciomed.eusal.es.

Vandaele, S. \& Gingras Harvey, M. Les nomenclatures anatomiques: histoire et traduction. Panacea, 14(38):222-34, 2013.

Vargas, C. A.; Ottone, N. E.; Contreras, M. \& del Sol. M. ¿Facies or Impressio in the Spleen? Int. J. Morphol., 34(3):1002-8, 2016.

Vásquez, B. \& del Sol, M. The Terminologia Histologica in the medical sciences. Int. J. Morphol., 32(1):375-80, 2014.

Vogl, A. W. Awareness of and access to a unified terminology by anatomists. Anat. Sci. Educ., 2(3):139-40, 2009.

Dirección para correspondencia:

Francisco Pérez Rojas

Escuela de Medicina

Universidad Autónoma de Chile

5 Poniente 1670

Talca

CHILE

Email: fperezr@uautonoma.cl

Recibido : 09-08-2017

Aceptado: 27-12-2017 